\title{
Residual-Based Discretization Error Estimation for Unsteady Flows
}

\author{
Tejaswini Gautham
}

\begin{abstract}
Thesis submitted to the Faculty of the Virginia Polytechnic Institute and State University in partial fulfillment of the requirements for the degree of

Master of Science

in

Aerospace Engineering
\end{abstract}

Christopher J. Roy, Chair

Jeff T. Borggaard

Heng Xiao

December 9, 2019

Blacksburg, Virginia

Keywords: CFD, truncation error, discretization error Copyright 2020, Tejaswini Gautham 


\section{Residual-Based Discretization Error Estimation for Unsteady Flows}

Tejaswini Gautham

\section{(ABSTRACT)}

Computational fluid dynamics (CFD) is a tool that is widely used in most industries today. It is important to have rigorous techniques to estimate the error produced when using CFD. This thesis develops techniques to estimate discretization error for unsteady flows using the unsteady error transport equation (ETE) as well as defect correction. A framework to obtain exact truncation error and estimated truncation error is also presented. The technique and results for the steady-state cases are given and the algorithm used for the steady case is extended for the unsteady case. Numerical results are presented for the steady viscous Burgers' equation, unsteady viscous Burgers' equation, steady quasi-1D Euler equations, and unsteady 1D Euler equations when applied to a shock tube. Cases using either defect correction or ETE are shown to give higher orders of accuracy for the corrected discretization error estimates when compared to the discretization error of the primal solution. 


\section{Residual-Based Discretization Error Estimation for Unsteady Flows}

Tejaswini Gautham

(GENERAL AUDIENCE ABSTRACT)

Computational fluid dynamics (CFD) is a tool that is widely used in most industries today. It is used to understand complex flows that are difficult to replicate using experimental techniques or by theoretical methods. It is important to have rigorous techniques to estimate the error produced when using CFD even when the exact solution is not available for comparison. This paper develops techniques to estimate discretization error for unsteady flows. Discretization error has one of the largest error magnitudes in CFD solutions. The exact physics dictates the use of continuous equations but to apply CFD techniques, the continuous equations have to be converted to discrete equations. Truncation error is, the error obtained when converting the continuous equations to discrete equations. This truncation error is in turn, the local source term for discretization error. To reduce the discretization error in the discrete equations, the exact or estimated truncation error is either added as a source term to the discrete equations or is used along with the error transport equation to get a better estimate of the solutions. A framework to obtain exact truncation error and estimated truncation error is also presented. The framework is first applied to the steady equations and is verified with results from previous studies and is then extended to the unsteady flows. 


\section{Dedication}

To my parents, Mr.Gautham and Mrs.Gautham 


\section{Acknowledgments}

Firstly, I would like to thank God for his constant support in all my endeavors. He gave me the strength to leave my country to pursue my dreams and to never give up no matter how hard times were.

I thank my research advisor, Dr. Christopher Roy, for always being available and guiding me throughout this work. I have learned so much about how research is conducted and how important it is to verify any solution you get.

I would like to thank the Physics Department for funding most of my master's degree. Not having to worry about money helped me conduct my research better.

I thank my committee members, Dr. Jeff Borggaard and Dr. Heng Xiao, for agreeing to serve on my committee and for offering their valuable insight.

I would like to also thank Hongyu Wang for all the help he has given me to successfully finish this work. Your insights were always very valuable.

I would like to also thank Will Tyson, Chip Jackson, and Weicheng Xue for helping me with any issues I had with my research and for constantly motivating me to do well.

I would also like to thank the staff in Randolph hall for making my life easier. Special thanks to Kelsey Wall and Erin Wilson for all their help over the years and Steve Edwards and Jonathan Spence for setting up my work station.

Lastly, I would like to thank my parents, my family in California, and my friends (Shruti and Abhinuv especially) for their unrelenting support and encouragement. 


\section{Contents}

List of Figures

viii

1 Introduction 1

1.1 Previous Work . . . . . . . . . . . . . . . . . . 2

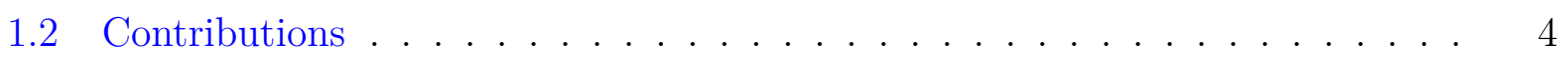

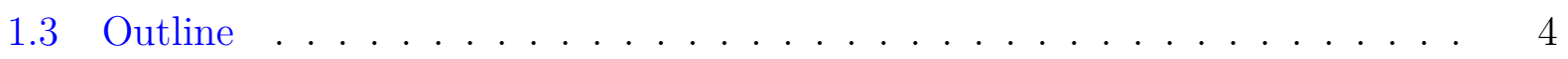

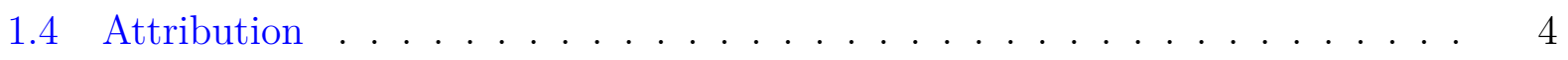

2 Discretization Error Estimation for Unsteady flows using ETE and defect $\begin{array}{lc}\text { correction } & 6\end{array}$

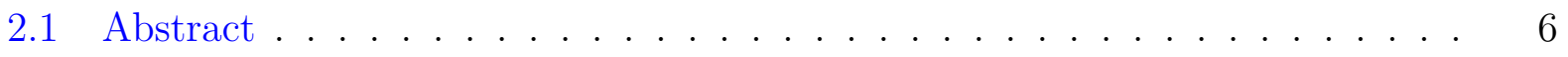

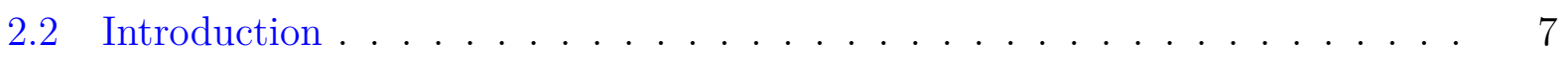

2.3 Governing Equations . . . . . . . . . . . . . . . . . 9

2.3 .1 Burgers' Equations . . . . . . . . . . . . . . . . . 9

2.3 .2 1D Euler Equations $\ldots \ldots \ldots \ldots$

2.4 Discretization Schemes and Truncation Error f . . . . . . . . . 15

2.4.1 Finite-Difference Discretization . . . . . . . . . . . . . 15

2.4 .2 Finite-Volume Discretization $\ldots \ldots \ldots \ldots \ldots$ 
2.4 .3 Explicit Euler . . . . . . . . . . . . . . . . . . . . . . . . . 17

2.4.4 Runge Kutta $2 / 4 \ldots \ldots \ldots$

2.4.5 Truncation Error . . . . . . . . . . . . . . . . . . . . . . . . 19

2.5 Order of Accuracy Analysis . . . . . . . . . . . . . . . . . . . . 21

2.6 Discretization Error Estimation Approaches . . . . . . . . . . . . . . . . 23

2.6 .1 Defect Correction . . . . . . . . . . . . . . . . . . . . 23

2.6.2 Non-Linear Error Transport Equation . . . . . . . . . . . . . . . 24

2.6.3 Linearized Steady Error Transport Equation . . . . . . . . . . . . . 25

2.7 Results \& Discussion . . . . . . . . . . . . . . . . . . . . . . . . . . . . . . . . 27

2.7.1 Burgers' Steady Results . . . . . . . . . . . . . . . . . . . . . 27

2.7 .2 Burgers' Unsteady Results . . . . . . . . . . . . . . . . . . . . . 30

2.7.3 Euler Steady Results . . . . . . . . . . . . . . . . . . . . . . 33

2.7.4 Euler Unsteady Results . . . . . . . . . . . . . . . . . . . . . 34

2.8 Conclusions $\ldots \ldots \ldots \ldots \ldots$

2.9 Acknowledgments . . . . . . . . . . . . . . . . . . . . . . . . . . . . . . . . 39

3 Discussion and Conclusions $\quad 40$

3.1 Future Work . . . . . . . . . . . . . . . . . . . . . . . . 41

$\begin{array}{ll}\text { Bibliography } & 42\end{array}$ 


\section{List of Figures}

2.1 Burgers' equation exact solution . . . . . . . . . . . . . . . . . . . 10

2.2 Area and Mach number distribution along the nozzle $\ldots \ldots \ldots$

2.3 Unsteady exact solution for density from $t=0.15$ s to $t=0.55 \mathrm{~s} \ldots \ldots 14$

2.4 Unsteady exact solution for velocity from $t=0.15 \mathrm{~s}$ to $t=0.55 \mathrm{~s} \ldots \ldots 14$

2.5 Unsteady exact solution for pressure from $t=0.15 \mathrm{~s}$ to $\mathrm{t}=0.55 \mathrm{~s} \ldots \ldots 15$

2.6 Steady Burgers' discretization error comparison with exact truncation error . 28

2.7 Steady Burgers' order analysis with exact truncation error . . . . . . . . 28

2.8 Steady Burgers' discretization error comparison with estimated truncation error 29

2.9 Steady Burgers' order analysis with estimated truncation error . . . . . . . 29

2.10 Unsteady Burgers' order analysis with exact truncation error . . . . . . . . 30

2.11 Unsteady Burgers' discretization error comparison with exact truncation error 31

2.12 Unsteady Burgers' discretization error comparison with estimated truncation

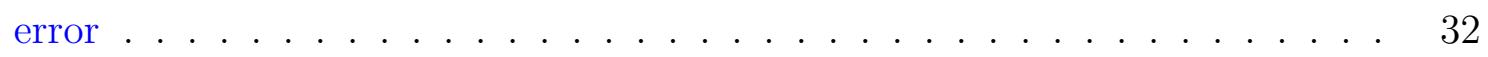

2.13 Unsteady Burgers' order analysis with estimated truncation error . . . . . . 32

2.14 Steady Euler discretization error comparison with exact truncation error . . 33

2.15 Steady Euler discretization error comparison with estimated truncation error 34

2.16 Steady Euler order analysis with estimated truncation error $\ldots . . . . . .34$ 
2.17 Unsteady Euler discretization error comparison with exact truncation error .

2.18 Unsteady Euler order analysis with exact truncation error . . . . . . . . 36

2.19 Unsteady Euler order analysis with estimated truncation error . . . . . . . 37

2.20 Unsteady Euler order analysis with estimated truncation error . . . . . . . . 37 


\section{List of Abbreviations}

\begin{tabular}{|c|c|}
\hline$\epsilon$ & Discretization Error \\
\hline$\nu$ & Viscosity \\
\hline$\tau$ & Truncation Error \\
\hline$\tilde{U}$ & Exact Solution \\
\hline$L$ & Partial Differential Equation \\
\hline$L_{h}$ & Discrete Equation \\
\hline$R$ & Residual \\
\hline$R e$ & Reynolds Number \\
\hline$t$ & Time \\
\hline$U_{h}$ & Discrete Solution \\
\hline$x$ & Position \\
\hline $\mathrm{d} t$ & time step \\
\hline
\end{tabular}




\section{Chapter 1}

\section{Introduction}

With the advent of high-speed computers, Computational fluid dynamics has become the norm in the Aerospace industry. Many real-world conditions are difficult to replicate and test and thus computational fluid dynamics can be used to understand complex physics. With the switch of a button, physical behavior can be turned on and off and various different cases can be analyzed. With increased reliance on CFD, the importance to understand the error that one can expect from CFD simulations has increased as well. The two main kinds of errors are modelling errors and numerical errors with numerical errors being the focus of this manuscript. Knowing the numerical error, will help us determine the accuracy of our solution and can also help us make corrections to our solution. In fields like nuclear engineering and aerospace engineering, even a tiny error of $0.1 \%$ can make a huge amount of difference and this is why one must understand both the source of the errors and the ways to reduce them.

Numerical errors can be further classified into round-off error, iterative error and discretization error. Converging the numerical solution down to machine precision and using double precision is often the best way to make round off error and iterative error negligible. There is no such easy solution for discretization error and thus it is the hardest and the most expensive kind of error to estimate. The value of the discretization error depends on the discretization scheme and the geometry defined. It is formally defined as the difference between the exact solution to the discrete equations and the exact solution to the partial differential 
equations. Discretization error is introduced locally into the solution by truncation error. Truncation error represents the higher-order terms which are truncated from Taylor's series expansions of the difference between the discrete and partial differential equations during the discretization process. In most practical cases, the exact solution is not known and the precise discretization error cannot be determined. Instead, the discretization error is estimated. There are many numerical techniques to estimate discretization error estimates and a comprehensive review of the different techniques out there can be found in [19].

It will also be beneficial to dwell on the importance of developing techniques to understand unsteady equations. A lot of work has been dedicated to the steady analysis of error in equations but not so much has been done for the unsteady flows. This added dimension makes the problems much larger and thus requires a lot more memory than the steady problems. Additionally, the easiest way to reduce discretization error is uniform grid refinement. But this only makes the massive unsteady problems only more difficult to solve. Thus, the primary focus of this work is to develop techniques using defect correction and ETE with finite difference discretization and finite volume discretization to estimation discretization error efficiently for unsteady equations.

\subsection{Previous Work}

A lot of literature has been dedicated to the development of defect correction. Fox [3] first introduced this technique and categorized it as a relaxation method that could be operated on ODEs and PDEs. Later Pereyra [11] [12] [10] made improvements on it and went on

to deal with more complex scenarios like nonlinear ODEs. Zadunaisky [27], applied it to a system of ODEs and Stetter [22], discussed how one could update and choose different defect correction functions with a rigorous mathematical derivation. Skeel [21] compares 
it with other techniques that are used to estimate global discretization error. Layton et al. [7] were one of the first to apply defect correction to an aerospace centric application. He applied it to the incompressible Navier-Stokes equations and got higher-order error estimates. Kurzen et al. [6] applied defect correction in his method of nearby problems. Naumovich et al. [8] used defect correction with algebraic multi grids for Linearized Euler equations. He computed first-order numerical solutions and used defect correction to correct the solution to second-order accuracy. Philips and Roy [14] applied it to various benchmark solutions and compared it with the results obtained from the error transport equation. Usage of defect correction for unsteady flow problems is relatively new and some of its findings are outlined in this manuscript.

A more recent approach to discretization error analysis is the Error Transport equation (ETE). ETE evaluates the propagation of discretization error over space and time. The source term for ETE is the truncation error and it can be either exact truncation error or estimated truncation error. Philips and Roy [13] [14] derived the truncation error to be in source term for ETE using the Generalized Truncation Error Expression (GTEE). They further show a way to linearize the steady ETE and thereby solve it implicitly. Tyson et al [25] [24] defines a novel reconstruction technique that could be used to find estimated truncation error and also compares the results from ETE to adjoint methods. Zhang et al. [28] used ETE to predict the location and magnitude of discretization error. Qin and Shih [15] discuss the propagation of discretization error using discrete ETE. Unsteady ETE is a relatively uncharted territory. The behavior of the nonlinear unsteady error transport equation was explored by Banks et al. [1] as it was applied to Hyperbolic PDEs and some of its key properties were outlined. Yan [26] applied the nonlinear ETE to unstructured finite-volume methods for unsteady problems. The unsteady ETE is an exciting new arena for exploration and some of its findings have been documented in this paper. 


\subsection{Contributions}

Error Transport Equation and defect correction is applied to two sets of governing equations for both steady and unsteady cases and a significant reduction in discretization error is seen. A framework to obtain exact truncation error and estimated truncation error is presented. The challenges of mixed order schemes and reconstruction in both space and time is also discussed.

\subsection{Outline}

The first chapter provides the reader with background information regarding discretization error, ETE, defect correction, and previous work in this area.

The second chapter reviews the effectiveness of using ETE and defect correction for reducing discretization error in numerical solutions and discusses the results of the two test cases. The third chapter offers a discussion of the work, draws conclusions, and provides areas for future improvement.

\subsection{Attribution}

As this thesis is written in a manuscript format, Chapter 2 of this work has multiple authors. To outline the contributions of each author, consider the following:

- Tejaswini Gautham (First Author): The first author served as the main contributor and the primary author of the work. The first author implemented a code that solves Burgers' equation and made changes to the steady flow solver so that it can handle unsteady flows. The first author has added in multiple subroutines to the primal Euler equations solver and 
has verified its ability to solve both steady and unsteady ETE and defect correction

- Hongyu Wang (Second Author): The second author provided guidance on different techniques that could be used to solve any problem that was encountered and provided helpful comments when drafting the manuscript for the work.

- Christopher J. Roy (Third Author): The third author provided valuable guidance and insight throughout the work and also provided helpful comments when drafting the manuscript. 


\section{Chapter 2}

\section{Discretization Error Estimation for}

\section{Unsteady flows using ETE and defect}

\section{correction}

Tejaswini Gautham ${ }^{1}$, Hongyu Wang ${ }^{2}$, Christopher J. Roy ${ }^{3}$

\section{$2.1 \quad$ Abstract}

Computational fluid dynamics (CFD) is a tool that is widely used in most industries today. In order to prove the credibility of CFD techniques, it is important to have estimates of the numerical error produced while using it. This paper develops techniques to estimate discretization error (generally the largest component of the numerical error) for unsteady flows using the unsteady Error Transport Equation(ETE) as well as defect correction. A framework to obtain exact truncation error and estimated truncation error is also presented. The technique and results for the steady state cases are also given and the algorithm used

\footnotetext{
${ }^{1}$ Graduate Student, Kevin T. Crofton Department of Aerospace and Ocean Engineering, 215 Randolph Hall.

${ }^{2}$ Graduate Student, Kevin T. Crofton Department of Aerospace and Ocean Engineering, 215 Randolph Hall.

${ }^{3}$ Professor, Kevin T. Crofton Department of Aerospace and Ocean Engineering, 215 Randolph Hall, AIAA Associate Fellow.
} 
for the steady case is extended to the unsteady case. Numerical results are presented for the steady viscous Burgers' equation, unsteady viscous Burgers' equation, steady quasi-1D nozzle flow and the unsteady 1D Euler equations when applied to a shock tube. Cases using either defect correction or ETE are shown to give higher-order discretization error estimates for the corrected discretization error when compared to the discretization error of the primal solution.

\subsection{Introduction}

With the advent of high-speed computers, CFD has become an indispensable tool in the Aerospace industry. Many real-world conditions are difficult to replicate and test and thus the usage of CFD to understand complex physics has become more commonplace. With the switch of a button, physical behavior can be turned on and off and various different cases can be analyzed. With increased reliance on $\mathrm{CFD}$, the importance to understand the error that one can expect from CFD simulations has increased as well. The two main kinds of errors are modelling errors and numerical errors with numerical errors being the focus of this paper [9]. Knowing the numerical error, will help us determine the accuracy of our solution and can also help us make corrections to our solution. In fields like nuclear engineering and aerospace engineering, even a tiny error can make a big impact on the performance of the system and this is why one must understand both the source of the errors and the ways to reduce them.

Numerical errors can be further classified into round-off error, iterative error and discretization error. Converging the numerical solution down to machine precision and using double precision is often the best way to make round off error and iterative error negligible. There is no such easy solution for discretization error and thus it is the hardest and the most ex- 
Chapter 2. Discretization Error Estimation for Unsteady flows using ETE and defect Tejaswini Gautham

Chapter 2

correction

pensive kind of error to estimate. It is formally defined as the difference between the exact solution to the discrete equations and the exact solution to the partial differential equations. There are many numerical techniques to estimate discretization error and most of them require solving the discrete equations on multiple grid levels. One such popular method is Richardson extrapolation [16] and it has been widely used in CFD literature. Even though it is an easy to implement post-processing method, this is very expensive in terms of computational resources because it needs the solution to be evaluated at multiple mesh levels and as we increase the dimensions in our problems, it becomes very cumbersome.

Alternatively, defect correction $[3,4,10,12,14,22]$ can be used to obtain an error estimate by re-solving the governing equations with the truncation error estimate added as a source term. In this way, the discrete solution is driven toward the exact solution to the partial differential equations (PDEs). This technique is explored in this paper and is shown to work well on both the unsteady and steady cases. A more recent approach to discretization error analysis is the Error Transport equation (ETE) [2, 15, 24, 28]. ETE evaluates the propagation of discretization error over space and time and is implemented here for both the unsteady and steady equations. The source term for ETE is the truncation error and the application of the exact truncation error and estimated truncation error have been explored.

The focus of this paper is to develop techniques to reduce error in unsteady flows and hence it will be beneficial to delve into the importance of this work. A lot of work has been dedicated to analysis of error in steady problems but not so much has been done for the unsteady case $[1,26]$ and particularly for finite difference schemes. This added dimension makes the problems larger and thus requires more memory than the steady problems. Thus, in this paper, techniques have been developed using defect correction and ETE using a finite difference discretization scheme for Burgers' and a finite volume discretization scheme for Euler equations to estimate discretization error, in an efficient manner, for unsteady 
equations.

In this paper, the discretization error is estimated for steady and unsteady flows using defect correction and ETE. The governing equations are discussed in section III, the discretization schemes and truncation error are discussed in section IV, the order of accuracy analysis is discussed in section $\mathrm{V}$, the methodology for both defect correction and ETE is given in section VI, the results are discussed in section VII and the conclusion in section VIII.

\subsection{Governing Equations}

\subsubsection{Burgers' Equations}

Burgers' equation is a quasi-linear parabolic partial differential equation and is shown in Eq. (2.1). Burgers' equation was chosen as the governing equation because of its simplicity, because it has multiple exact solutions and because it is a scalar model equation for the Navier-Stokes equations. The viscous shock solution was chosen for this analysis because it can be correlated to a real phenomenon and because it is smooth and non-trivial. For the steady case, the time-dependent term does not impact the final solution and is only used for pseudo time stepping.

$$
\frac{\partial u}{\partial t}+u \frac{\partial u}{\partial x}=\nu \frac{\partial^{2} u}{\partial x^{2}}
$$

Burger's equation can be re-written in conservation form as:

$$
\frac{\partial u}{\partial t}+\frac{\partial u^{2} / 2}{\partial x}=\nu \frac{\partial^{2} u}{\partial x^{2}}
$$


Chapter 2. Discretization Error Estimation for Unsteady flows using ETE and defect Tejaswini Gautham

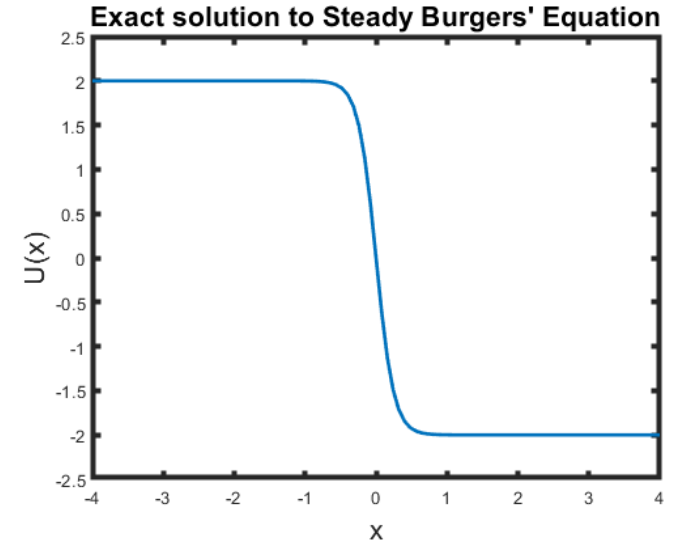

(a) Steady Burgers' viscous shock exact solution

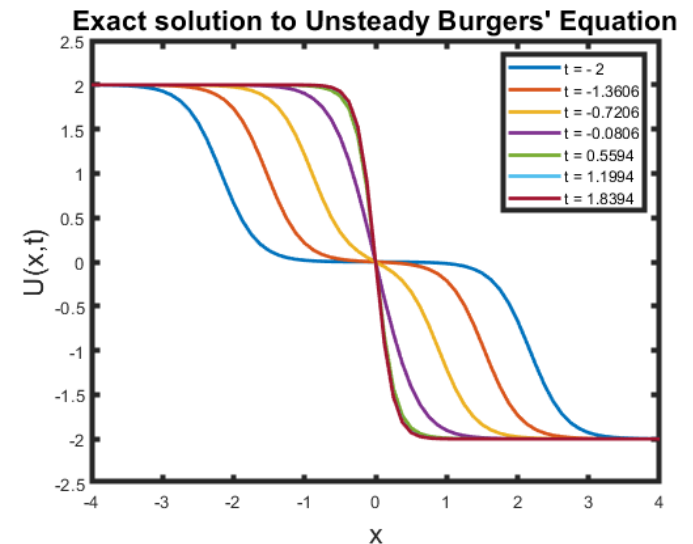

(b) Unsteady Burgers' viscous shock exact solution

Figure 2.1: Burgers' equation exact solution

The exact steady viscous shock solution is shown in Figure 2.1a and the equation is given as

$$
u=\frac{-2 \alpha \nu}{L} \tanh \left(\frac{\alpha x}{L}\right)
$$

The exact unsteady viscous shock solution is shown in Figure 2.1b and the equation is given as

$$
u=\frac{-2 \alpha \nu}{L} \frac{\sinh \left(\frac{\alpha x}{L}\right)}{\cosh \left(\frac{\alpha x}{L}\right)+\exp \left(\frac{-\alpha^{2} \nu t}{L^{2}}\right)}
$$

Where $\alpha$ is the scaling factor which is taken as half the Reynolds number, Reynolds number is selected to be 64 and $\mathrm{L}$ is the length of the domain and is taken to be 8 . The range of spatial values is taken to be $-\mathrm{L} / 2$ to $\mathrm{L} / 2$ and the range of temporal values is taken to be -2 to 2 .

\subsubsection{D Euler Equations}

The Euler equations are a combination of the conservation of mass, momentum and energy of a fluid. The unsteady quasi-1D Euler equations in strong, conservation law form are 


$$
\frac{\partial A u}{\partial t}+\frac{\partial A F(u)}{\partial x}=s(u)
$$

where $\mathrm{u}$ is the vector of conserved variables, $\mathrm{F}(\mathrm{u})$ is the vector of inviscid fluxes, $\mathrm{A}$ is the area and $\mathrm{s}(\mathrm{u})$ is a source term. The conserved variables, inviscid fluxes, and source term are given by

$$
u=\left[\begin{array}{c}
\rho \\
\rho u \\
\rho e_{t}
\end{array}\right] \quad F(u)=\left[\begin{array}{c}
\rho u \\
\rho u^{2}+P \\
\rho u h_{t}
\end{array}\right] \quad s(u)=\left[\begin{array}{c}
0 \\
P \frac{d A}{d x} \\
0
\end{array}\right]
$$

where $\rho$ is the fluid density, $\mathrm{u}$ is the fluid, $\mathrm{p}$ is the static pressure, $e_{t}$ is the total energy, and $h_{t}$ is the total enthalpy. The system of equations is closed by using the equation of state for a perfect gas such that the total energy and total enthalpy are given by

$$
e_{t}=\frac{P}{\rho(\gamma-1)}+\frac{u^{2}}{2} \quad h_{t}=\frac{\gamma P}{\rho(\gamma-1)}+\frac{u^{2}}{2}
$$

where $\gamma=1.4$ is the ratio of the specific heats for air.

For the steady case, we consider a converging diverging nozzle that has a Gaussian area distribution defined by Eq. (2.8) and is shown in Figure 2.2. The constant $\sigma$ is taken to be 0.2 for our case.

$$
A(x)=1-0.8 e^{\frac{-x^{2}}{2 \sigma^{2}}}, \quad x \in[-1,1]
$$

The nozzle is characterized by a contraction through which the flow is accelerated followed by a diverging section where the flow expands supersonically as defined by the pressure set at the exit and is shown in Figure 2.2. An exact solution is obtained using the following 
Chapter 2. Discretization Error Estimation for Unsteady flows using ETE and defect Tejaswini Gautham

Area-Mach number relationship

$$
\frac{A(x)}{A^{*}}=\frac{1}{M^{2}}\left[\frac{2}{\gamma+1}\left(1+\frac{\gamma-1}{2} M^{2}\right)\right]^{\frac{\gamma+1}{\gamma-1}}
$$

where $\mathrm{A}(\mathrm{x})$ is the cross-sectional area and $A^{*}$ is the cross-sectional area when Mach number is one. Once the local Mach number is calculated, the other primitive variables can be solved using the stagnation conditions. For the boundary conditions, the inflow Mach number is extrapolated from the interior to set the value of the boundary face. At the outflow face, all primitive variables are extrapolated from the interior to set the value of the outflow face.

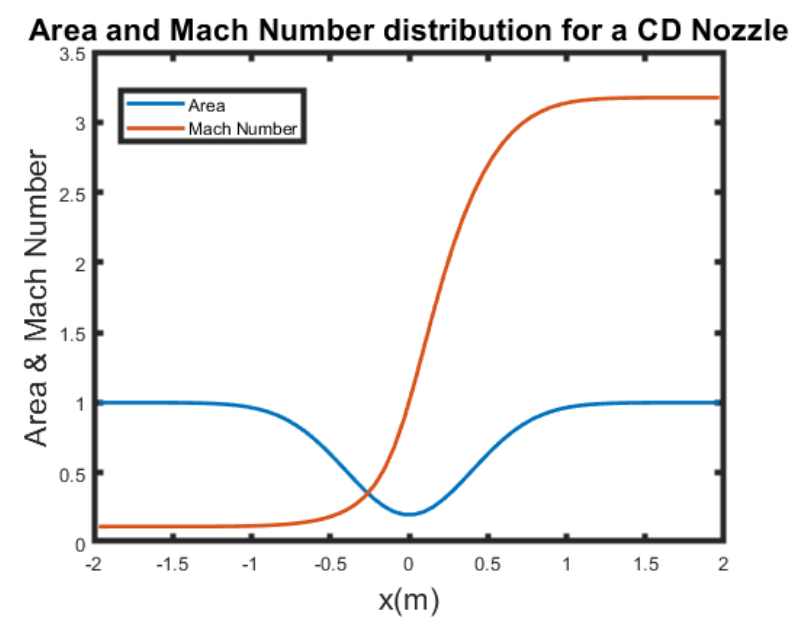

Figure 2.2: Area and Mach number distribution along the nozzle

The unsteady 1D Euler equations are used to solve for the flow in a shock tube. For this case, the area is taken to be constant and all area derivatives are taken to be zero. With regard to an exact solution for the unsteady Euler equations, first a manufactured solution is used as it is a smooth solution and it can be used to analyze any coding bugs in a rigorous manner [17]. It has the following form

$$
\rho=\rho_{0}+\rho_{x} \sin \left(\frac{a_{r x} \pi x}{L}\right)+\rho_{t} \cos \left(\frac{a_{r t} \pi t}{T}\right)
$$




$$
\begin{aligned}
& u=u_{0}+u_{x} \sin \left(\frac{a_{u x} \pi x}{L}\right)+u_{t} \cos \left(\frac{a_{u t} \pi t}{T}\right) \\
& P=p_{0}+p_{x} \sin \left(\frac{a_{p x} \pi x}{L}\right)+p_{t} \cos \left(\frac{a_{p t} \pi t}{T}\right)
\end{aligned}
$$

where $\mathrm{L}$ is the length of the domain, $\mathrm{T}$ is the total time being analyzed, $\mathrm{x}$ is the spatial location being analyzed and $\mathrm{t}$ is the instant in time being analyzed. The constants in the MMS solution are chosen such that all the terms in the solution are appropriately exercised. In this case, $u_{0}$ is taken to be $100, u_{x}$ and $u_{t}$ are $25, \rho_{0}$ is $1, \rho_{x}$ and $\rho_{t}$ are $0.25, P_{0}$ is 1e5, $P_{x}$ and $P_{t}$ are 25,000. All the amplitude terms are fractions between zero and one.

The exact solution for the unsteady Euler equations is chosen to be the exact solution of the Riemann problem [23]. The solution represents a system of hyperbolic conservation laws subject to the simplest, non-trivial, initial conditions. In spite of its simplicity, the initial data for the solution of the Riemann problem contains the fundamental physical and mathematical character of the relevant set of conservation laws and is therefore a valuable tool to have. It has multiple solutions, based on its initial conditions, and each of them include a combination of expansion fans and/or shock waves. The case that has been chosen for analysis in this paper is the shock tube problem. It is a special case of the general Riemann problem where the initial velocities at both ends of the tube are zero. A diaphragm is placed at the center of the tube. At time $=0$, the diaphragm is broken and the simulation develops over time with a expansion fan to the left of the original diaphragm and a shock wave to the right of it. The figures below show the density, velocity and pressure across the length of the tube for time $=0.15 \mathrm{~s}$ to time $=0.55 \mathrm{~s}$ after the diaphragm is broken. 
Chapter 2. Discretization Error Estimation for Unsteady flows using ETE and defect Tejaswini Gautham

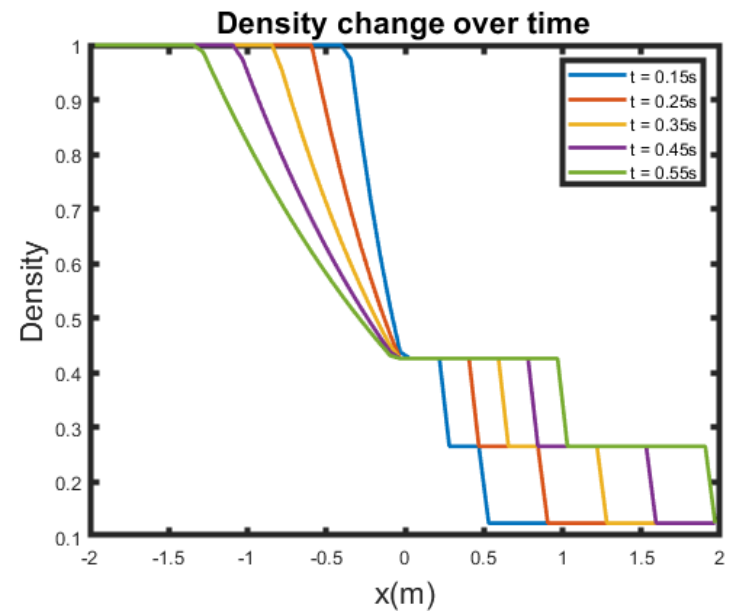

Figure 2.3: Unsteady exact solution for density from $\mathrm{t}=0.15 \mathrm{~s}$ to $\mathrm{t}=0.55 \mathrm{~s}$

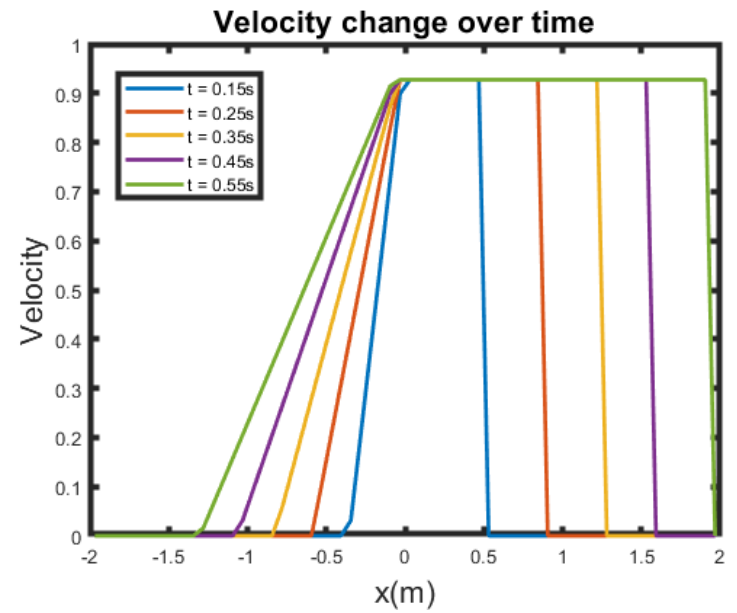

Figure 2.4: Unsteady exact solution for velocity from $t=0.15 \mathrm{~s}$ to $t=0.55 \mathrm{~s}$ 


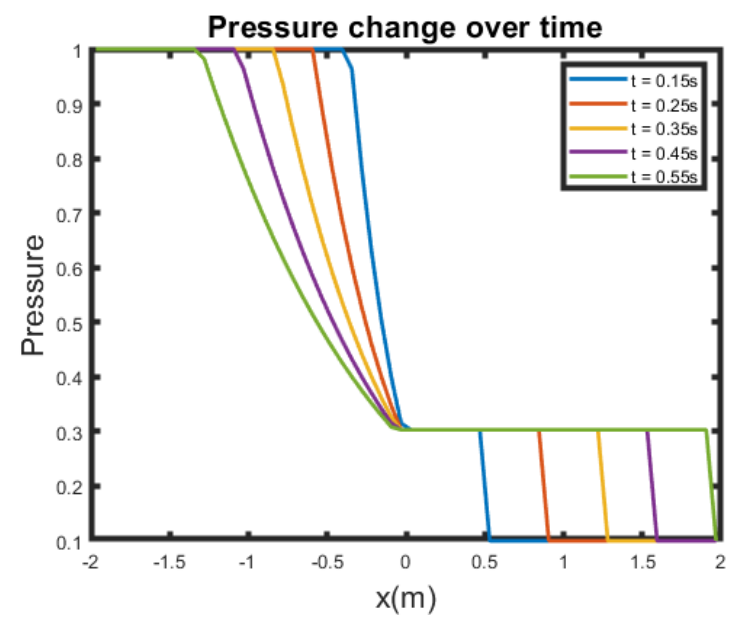

Figure 2.5: Unsteady exact solution for pressure from $\mathrm{t}=0.15 \mathrm{~s}$ to $\mathrm{t}=0.55 \mathrm{~s}$

\subsection{Discretization Schemes and Truncation Error}

The governing equations were discretized in time on a uniform grid using explicit Euler for the steady equations and unsteady equations, and 2 step and 4 step Runge-Kutta for the unsteady equation. For the spatial discretization, two different schemes, finite-difference and finite-volume discretization, were used for Burgers' and the Euler equations, respectively. We also discuss truncation error in this section, which is used as a source term in the primal equations for defect correction and in the error transport equations to get higher-order discretization error estimates

\subsubsection{Finite-Difference Discretization}

The general principal of finite difference schemes is similar to that of using numerical schemes to solve ordinary differential equations. It consists of approximating the partial differential operator by replacing the derivatives in the equation with algebraic expressions. The domain is partitioned into temporal and spatial nodes and the approximations are computed using 
Chapter 2. Discretization Error Estimation for Unsteady flows using ETE and defect Tejaswini Gautham

Chapter 2

correction

the appropriate value of time and space for each node. The most common way to approximate the partial differences in finite-difference schemes is by using Taylor series expansions. For instance:

$$
u(i+1)=u(i)+\frac{\partial u}{\partial x}_{i} \Delta x+\frac{\partial^{2} u}{\partial x^{2}} \frac{(\Delta x)^{2}}{2}+O\left(\Delta x^{3}\right)
$$

Rearranging the above equation, we get our expression for the first derivative of $u$ as:

$$
\frac{\partial u}{\partial x}=\frac{u(i+1)-u(i)}{\Delta x}+O(\Delta x)
$$

Using the technique above, we get our spatial residual for Burgers' equation which is second order accurate.

$$
R(i, n)=\frac{\nu(u(i+1, n)-2 u(i, n)+u(i-1, n))}{\Delta x^{2}}-\frac{\frac{u^{2}(i+1, n)}{2}-\frac{u^{2}(i-1, n)}{2}}{2 \Delta x}
$$

\subsubsection{Finite-Volume Discretization}

The general conservation law is stated as:

$$
\frac{\partial \tilde{u}}{\partial t}+\nabla F(\tilde{u})=s(\tilde{u})
$$

defined over the domain $\Omega \subset \Re^{m} \times[0, \infty], x \in \Re^{m}, m \in\{1,2,3\}$ where $\tilde{u}$ are the conserved quantities, $\mathrm{F}()$ is the flux tensor, and $\mathrm{s}()$ is a source term. In general, the flux tensor and source term may also be functions of $\nabla \tilde{u}$, but this dependency has been omitted here for the sake of brevity. The solution domain, $\Omega$, is decomposed into a set of non-overlapping control volumes. The general conservation law may be cast into a weak form by integrating 
over each control volume, $\Omega_{i}$, and using the Gauss divergence theorem to convert the flux divergence integral into a surface integral. For a fixed control volume, the weak form is given as

$$
L(\tilde{u})=\frac{\partial}{\partial t} \int_{\Omega_{i}} \tilde{u} d \Omega+\int_{\partial \Omega_{i}} F(\tilde{u}) \cdot \hat{n} d S-\int_{\Omega_{i}} s(\tilde{u}) d \Omega=0
$$

where $\hat{n}$ is the outward unit normal vector on the surface of $\Omega_{i}$. In the finite-volume method, a discrete solution, $u_{h}$, is sought which approximates the control volume average of the exact continuous solution, $\tilde{u}$.

In our case, the spatial discretization is second order accurate, cell-centered and the fluxes are computed using Central flux scheme and the 2/4 Jameson Damping for stability [5].

\subsubsection{Explicit Euler}

Explicit Euler is a first order accurate time-integration scheme that is used to march the equations in time as shown in Eq.(2.18). After setting the boundary conditions from the exact solution, for the interior cells, we start with a random guess for the initial solution and the equations are marched in time until convergence is reached. In the unsteady case, we use the same equations but with an accurate initial condition which we get from the exact solution and march in time until the desired time is reached.

$$
u(i, n+1)=u(i, n)+\delta t R(i, n)
$$

where $\mathrm{i}$ represents the spatial node, $\mathrm{n}$ represents the temporal node and $\mathrm{R}$ is the spatial residual. 
Chapter 2. Discretization Error Estimation for Unsteady flows using ETE and defect Tejaswini Gautham

Chapter 2

correction

\subsubsection{Runge Kutta 2/4}

The Runge-Kutta methods are a family of single-step, explicit, numerical techniques for solving a first-order ordinary differential equations. They are ideal to solve unsteady partial differential equations as they have simple time-integration formulas that can be used to treat unsteady equations to the desired level of accuracy. In this paper, we use the 2 step RungeKutta method and the 4 step Runge-Kutta method. The 2 step Runge-Kutta is second order accurate in time. As seen in Eq. (2.19) and in Eq. (2.20), the residual is calculated at two time steps. These residuals are then combined to get a higher order solution as seen in Eq. (2.21). The 4 step Runge-Kutta is fourth order accurate in time and follows a similar

algorithm to that of the 2 step Runge-Kutta. As seen in Eq. (2.22) to Eq. (2.26), 4 step Runge-Kutta computes the residual at 4 time steps and then combines them to get a higher order solution.

For the 2 step Runge-Kutta Technique:

$$
\begin{gathered}
K 1=R\left(u^{n}, t^{n}\right) \\
K 2=R\left(u^{n}-\frac{K 1}{2}, t^{n}+\frac{\Delta t}{2}\right) \\
u(i, n+1)=u(i, n)-\Delta t K 2
\end{gathered}
$$

For the 4 step Runge-Kutta Technique:

$$
K 1=R\left(u^{n}, t^{n}\right)
$$




$$
\begin{gathered}
K 2=R\left(u^{n}-\frac{K 1}{2}, t^{n}+\frac{\Delta t}{2}\right) \\
K 3=R\left(u^{n}-\frac{K 2}{2}, t^{n}+\frac{\Delta t}{2}\right) \\
K 4=R\left(u^{n}-K 3, t^{n}+\Delta t\right) \\
u(i, n+1)=u(i, n)-\Delta t \frac{1}{6}(K 1+2 K 2+2 K 3+K 4)
\end{gathered}
$$

\subsubsection{Truncation Error}

Truncation Error is defined as the difference between the discrete equations and the partial differential equations. As truncation error acts as the source term to defect correction and ETE, we need to solve for it first. The numerical solution for the given partial differential equation can be represented using the General Truncation Error Expression (GTEE) [18].

$$
L_{h}(u)=L(u)+\tau_{h}(u)
$$

where $L_{h}$ represents the discretized governing equations and operates on a discrete space, $L$ represents the continuous governing equations and operates on a continuous space, and $\tau_{h}$ represents the truncation error.

\section{Exact Truncation Error}

We can insert the exact solution to the PDE into the above equation and we get an 
Chapter 2. Discretization Error Estimation for Unsteady flows using ETE and defect Tejaswini Gautham

Chapter 2

correction

expression for the exact truncation error Eq (2.28). Therefore, we plug in our exact solution into our discretization scheme to get our exact truncation error. For the steady exact truncation error, this is just the spatial residual obtained from plugging in the exact solution. For the unsteady truncation error, it is both the unsteady and steady residual at every time step.

$$
\tau_{h}(\tilde{u})=L_{h}(\tilde{u})
$$

\section{Estimated Truncation Error}

The exact solution is not generally known and in this case the truncation error must be estimated using the reconstructed discrete solution. First, the primal problem is solved and a discrete solution is obtained. This discrete solution is then prolonged to continuous space using a polynomial least-squares fit for finite difference methods (FDM) or k-exact reconstruction for finite volume methods (FVM)[25]. It is prolonged to a $\mathrm{k}$ order space (denoted by $I_{h}^{k}$ ) and it is then operated on by the continuous operator(the PDE) as shown in Eq. (2.29). For higher order reconstruction schemes, the data needs to be centered and scaled before the solution reconstruction is performed. Even after centering and scaling, if the reconstruction matrix is badly scaled, a singular-value decomposition is performed. For the steady case, a one-dimensional reconstruction in space was performed and for the unsteady case, a two-dimensional reconstruction in space and time is performed at every time step.

$$
\tau_{h}(\tilde{u}) \approx-I^{h} L\left(I_{h}^{k} u_{h}\right)
$$




\subsection{Order of Accuracy Analysis}

Order of accuracy is the rate at which the discrete solution approaches the exact solution to the PDEs. The order of accuracy test examines not only the convergence of the numerical solution but also if the discretization error is reduced at the expected rate as the spatial and/or time step is reduced. With regard to code verification, it is one of the hardest tests to satisfy and thus is very rigorous. The theoretical rate is called the formal order of accuracy and is found by carrying out the truncation error analysis on the discretization scheme using the Taylor series expansions. The actual rate at which the discretization error is reduced is called the observed order of accuracy and its calculation requires two systematically refined meshes when the exact solution is available. To analyze the steady and unsteady cases investigated in this paper, the order of accuracy formula is different for each case

In our steady analysis, the residual discretization scheme is second order accurate in space. The temporal accuracy is not of importance here and therefore, our only focus is spatial mesh refinement. We can use Eq. (2.30) to calculate the expected order of accuracy.

$$
\hat{p}=\frac{\ln \left(\frac{\left.\epsilon_{r h}\right)}{\left.\epsilon_{h}\right)}\right.}{\ln (r)} \quad r=\frac{h_{\text {coarse }}}{h_{\text {fine }}}
$$

In the above formula, $\mathrm{r}$ is the refinement factor and $\mathrm{h}$ is the spacing between two nodes in the mesh. While applying the explicit 2 step Runge-Kutta temporal scheme to unsteady equations with a second order spatial discretization scheme, the scheme is second order accurate in space and in the time and the overall order of accuracy is also second. In this case, we can refine in time and space by the same factor to get the observed order of accuracy.

$$
\hat{p}=\frac{\ln \left(\frac{\left.\epsilon_{r h}^{r t}\right)}{\left.\epsilon_{h}^{t}\right)}\right.}{\ln (r)}
$$


Chapter 2. Discretization Error Estimation for Unsteady flows using ETE and defect Tejaswini Gautham

Chapter 2

correction

While applying the explicit Euler temporal scheme to unsteady equations with a second order spatial scheme, which is first order in time and second order in space, we see that it gets complicated because it is now a mixed order scheme. The temporal error might be significantly higher than the spatial error or vice versa and this might not give us the order of accuracy we expect based on the theoretical order of accuracy. A good example of this case is Burgers' equation. While using explicit Euler discretization, we expect the overall order to be one. But because the temporal terms are very small compared to the spatial terms, the observed order of accuracy comes out to be two. This anomaly was further investigated using the technique below. The equation below is the general formulation for discretization error:

$$
\epsilon_{h x}^{h t}=g_{x} h_{x}^{p}+g_{t} h_{t}^{q}+g_{x t} h_{x}^{r} h_{t}^{s}
$$

In most cases, the mixed order term can be ignored [9] and we can calculate just the separate orders of accuracy, one in time and one in space using four mesh levels. The terms $g_{x}$ and $g_{t}$ give the magnitude of spatial and temporal errors respectively. If one is significantly bigger than the other, the observed order of accuracy will be not be the same as the formal order of accuracy. But in some cases, the mixed order term might be important and the separate order analysis might not give us any meaningful results [20]. In this case, the full non-linear equation must be solved in a least-squares sense to get all the seven unknowns. An example of this case is when estimated truncation error is applied to unsteady Burgers' equation. The temporal order is affected by the mixed order term as the mixed order errors and temporal errors are the same order of magnitude. Hence, solving the full-nonlinear equation will give us a better understanding of the order of accuracy. 


\subsection{Discretization Error Estimation Approaches}

The discretization error is the difference between the exact solution to the discrete equations and the exact solution to the partial differential equations and is shown in Eq. (2.33)

$$
\epsilon=u_{h}-I^{h} \tilde{u}
$$

There are two techniques that have been explored for steady and unsteady flows in this work. They both obtain higher orders of discretization error estimates in the solution and therefore can be used to correct the solution and obtain a better estimate of the solution. The advantages and accuracy of each of these techniques will be discussed in the results section.

\subsubsection{Defect Correction}

In defect correction, we improve the discretization error estimate obtained from solving the primal problem by re-solving the primal problem with a source term. This will give us the corrected solution of lower discretization error than the primal solution. In defect correction, the source term that is used is the truncation error and it is obtained as follows: First we get our expressions for truncation error. Plugging in $\tilde{u}$ into the GTEE expression:

$$
L_{h}(\tilde{u})=L(\tilde{u})+\tau(\tilde{u})
$$

But, $L(\tilde{u})=0$ because the exact solution is being plugged into the exact PDE

$$
L_{h}(\tilde{u})=\tau_{h}(\tilde{u})
$$


Chapter 2. Discretization Error Estimation for Unsteady flows using ETE and defect Tejaswini Gautham

Solving the above problem would give us:

$$
L_{h}\left(\overline{u_{h}}\right)=\tau(\tilde{u})
$$

where $\overline{u_{h}}$ is an approximation of $\tilde{u}$ that is higher order than $u_{h}$ Therefore, using exact truncation, we get:

$$
L_{h}\left(\overline{u_{h}}\right)=L_{h}\left(I^{h} \tilde{u}\right)
$$

For estimated truncation error, it is as follows:

$$
L_{h}\left(\overline{u_{h}}\right)=-I_{h} L\left(I_{h}^{k} u_{h}\right)
$$

Now that we have an expression for truncation error, we go back to our PDE expression and plug it in,

$$
L_{h}\left(u_{h}\right)=\frac{d u_{h}}{d t}+R_{h}\left(u_{h}\right)=0
$$

From the above truncation error expressions, we get our expression for defect correction as follow:

$$
\frac{d u_{h}}{d t}+R_{h}\left(u_{h}\right)=\tau
$$

where $\tau$ can be either exact or estimated truncation error. This expression is applicable for both the steady and unsteady defect correction. The only difference for the steady case is that, the first term will be set to zero.

\subsubsection{Non-Linear Error Transport Equation}

To derive the non-linear error transport equation, we start off with the GTEE. We insert the exact solution of the PDE into GTEE and subtract $L_{h}\left(u_{h}\right)$ from both sides and simplify 
to get:

$$
L_{h}\left(u_{h}-\epsilon_{h}\right)-L_{h}\left(u_{h}\right)=\tau
$$

Substituting the discrete PDE (as given in Eq. (2.39)) into it:

$$
-\frac{d u_{h}}{d t}+\frac{d \epsilon_{h}}{d t}-R_{h}\left(u_{h}-\epsilon_{h}\right)+\frac{d u_{h}}{d t}+R_{h}\left(u_{h}\right)=\tau
$$

Simplifying, we get Non-linear unsteady ETE:

$$
\frac{d \epsilon_{h}}{d t}-R_{h}\left(u_{h}-\epsilon_{h}\right)+R_{h}\left(u_{h}\right)=\tau
$$

For steady equations, $R_{h}\left(u_{h}\right)=0$, and it simplifies to:

$$
\frac{\partial \epsilon_{h}}{\partial t}-R\left(u_{h}-\epsilon_{h}\right)=-\tau
$$

The above equation is evolved in time using the same discretization scheme as the primal problem. The discretization error at the first-time step is taken to be zero and then time evolved from there. In all the above equations $\tau$ refers to truncation error and can be either exact or estimated truncation error and can be substituted to get the appropriate equation for each case.

\subsubsection{Linearized Steady Error Transport Equation}

To derive the steady non-linear error transport equation, we start off with Eq. (2.41) but $R_{h}\left(u_{h}\right)=0$ for steady problems. We simplify to get: 
Chapter 2. Discretization Error Estimation for Unsteady flows using ETE and defect Tejaswini Gautham

$$
L_{h}\left(\epsilon_{h}\right)=-\tau_{h}(\tilde{u})
$$

The above expression can be used if the discrete operator is linear. For a general system of PDEs, the linearization is found by expanding the discrete operator evaluated at the exact solution about the discrete operator evaluated using the numerical solution

$$
\begin{gathered}
L_{h}(\tilde{u})=L_{h}\left(u_{h}\right)-\epsilon_{h} \frac{\partial L_{h}}{\partial u}+\frac{\epsilon_{h}^{2}}{2} \frac{\partial^{2} L_{h}\left(u_{h}\right)}{\partial u^{2}}+O\left(\epsilon_{h}^{3}\right) \\
L_{h}\left(\epsilon_{h}\right)=L_{h}\left(u_{h}\right)-L_{h}(\tilde{u})=\epsilon_{h} \frac{\partial L_{h}\left(u_{h}\right)}{\partial u_{h}}-\frac{\epsilon_{h}^{2}}{2} \frac{\partial^{2} L_{h}\left(u_{h}\right)}{\partial u^{2}}+O\left(\epsilon_{h}^{3}\right)
\end{gathered}
$$

Neglecting higher order terms, this results in the linearized error transport equation

$$
\frac{\partial L_{h}\left(u_{h}\right)}{\partial u} \epsilon_{h}=-\tau_{h}\left(u_{h}\right)+O\left(\epsilon_{h}^{2}\right)
$$

If the Jacobian is directly solved, it might lead to issues because it might be badly conditioned. In that case, pseudo-time stepping can be used to get an accurate solution [25].

$$
\left(\Delta+\frac{\partial L_{h}\left(u_{h}\right)}{\partial u_{h}}\right) \Delta \epsilon_{h}=\tau_{h}+\frac{\partial L_{h}\left(u_{h}\right)}{\partial u_{h}} \epsilon_{h}(n)
$$

where $\Delta$ is any small number

$$
\epsilon_{h}(n+1)=\epsilon(n)+\Delta \epsilon
$$




\subsection{Results \& Discussion}

\subsubsection{Burgers' Steady Results}

Steady equations are analyzed first as there are well-documented results that it can be compared against before adding a layer of complexity arising from the unsteadiness of the equations. The steady primal problem is solved and its order is verified using the exact solution. After that, defect correction and linearized ETE are operated on the primal problem. For defect correction, first, the exact truncation error is plugged in as a source term in the discrete equations to get the new estimate of the solution for the primal problem. This new estimate is compared against the exact solution to obtain the corrected discretization error and as shown in Figure 2.6, this gives us a much lower error than the primal discretization error. In this case, an order analysis is not performed as the error goes to machine zero and therefore cannot be reduced even with mesh refinement. Similarly, estimated truncation error is plugged into the linearized steady ETE. The error estimate obtained from ETE is corrected with the discretization error from the primal problem to give us a corrected discretization error. This corrected discretization error is a fourth order accurate estimate as seen in Figure 2.7. In this case, defect correction is more expensive than ETE because defect correction resolves the entire primal problem with truncation error as the source term until a tolerance is reached while linearized ETE is solving a single linear system after the primal has been converged. 
Chapter 2. Discretization Error Estimation for Unsteady flows using ETE and defect Tejaswini Gautham

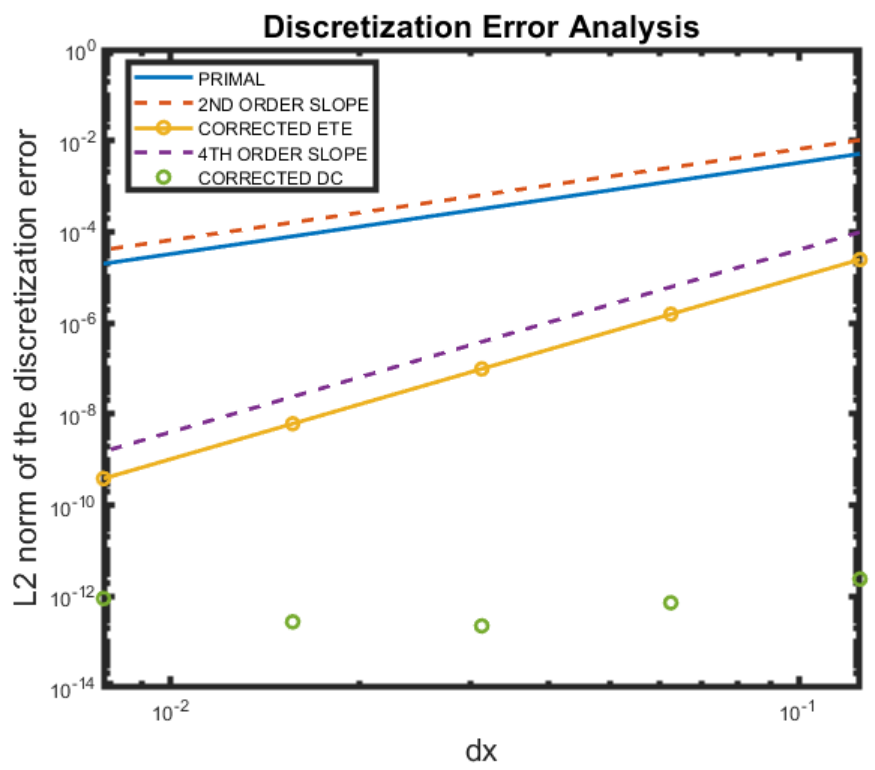

Figure 2.6: Steady Burgers' discretization error comparison with exact truncation error

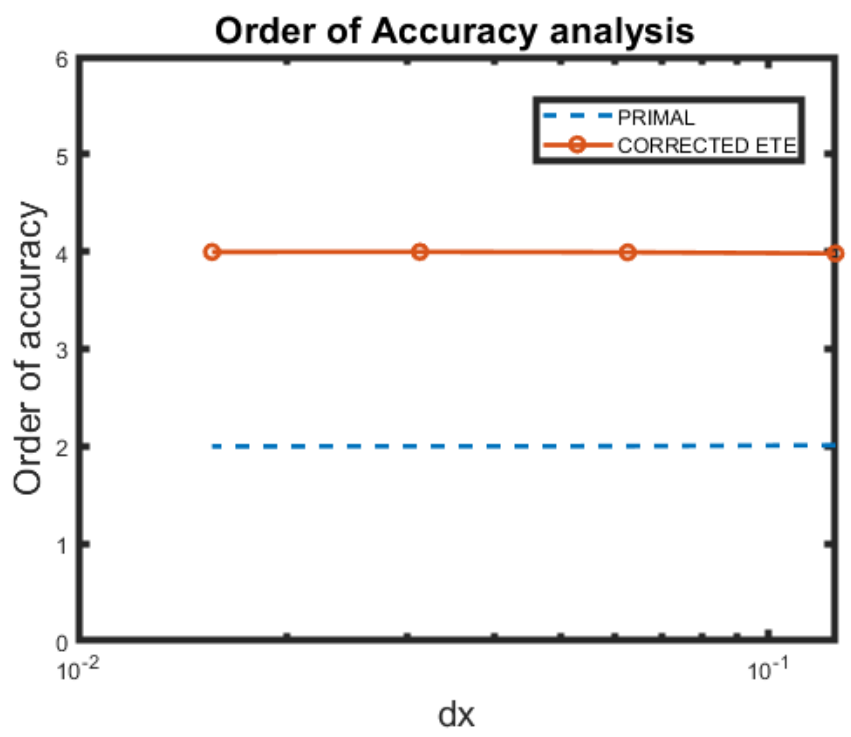

Figure 2.7: Steady Burgers' order analysis with exact truncation error

A similar approach can be adopted with the estimated truncation error for defect corrected and error transport equation and from Figures 2.8 and 2.9, we see that we get a fourth order accurate corrected discretization error for both defect correction and ETE in comparison 
to the second order accurate primal discretization error. A second order primal solution is brought to fourth order accuracy by adding a second order accurate truncation error. In the absence of an exact solution, we see that truncation error can be estimated in a rigorous manner to bring down the discretization error considerably.

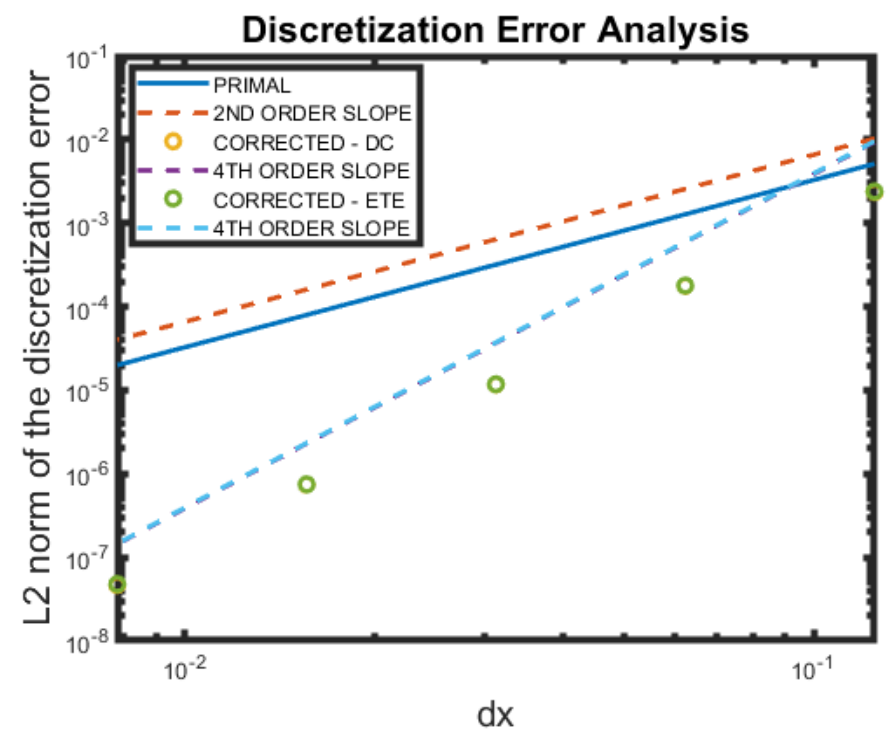

Figure 2.8: Steady Burgers' discretization error comparison with estimated truncation error

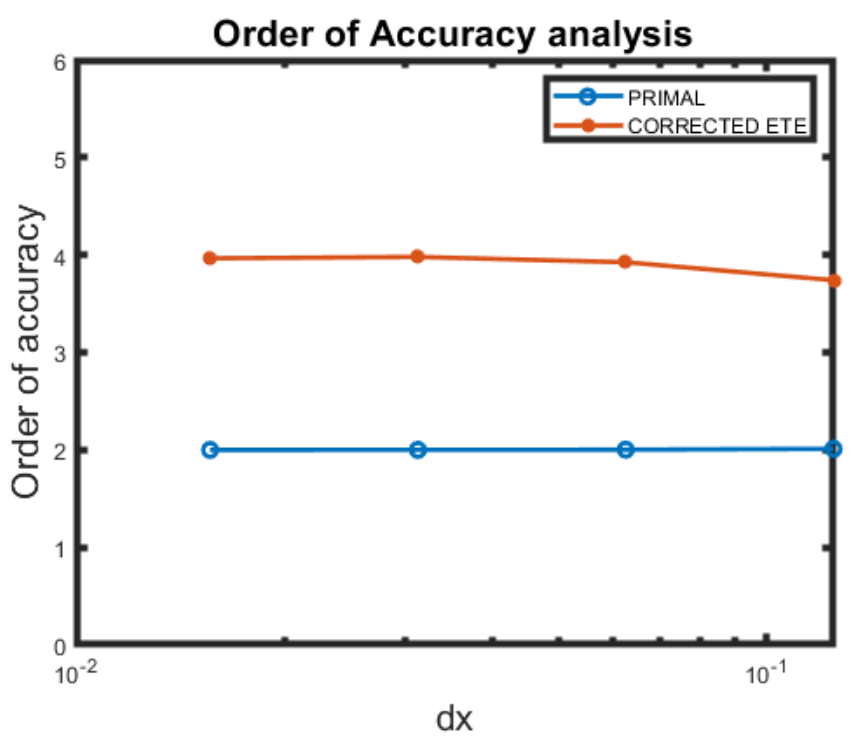

Figure 2.9: Steady Burgers' order analysis with estimated truncation error 
Chapter 2. Discretization Error Estimation for Unsteady flows using ETE and defect Tejaswini Gautham

Chapter 2

correction

\subsubsection{Burgers' Unsteady Results}

When the steady algorithm is extended for the unsteady case, the exact truncation error is computed and inserted as a source term into the discrete equations at each spatial and temporal location. As seen in Figure 2.10, this gives the corrected discretization error of exactly zero. Similarly, unsteady ETE is evolved over time with exact truncation error as the source term, and as seen in Figure 2.11, this gives us an error of machine precision. This extraordinary result is obtained because the exact truncation error drives the discrete solution to the exact solution. As we are using non-linear ETE in this case, the cost of defect correction versus ETE are along the same order.

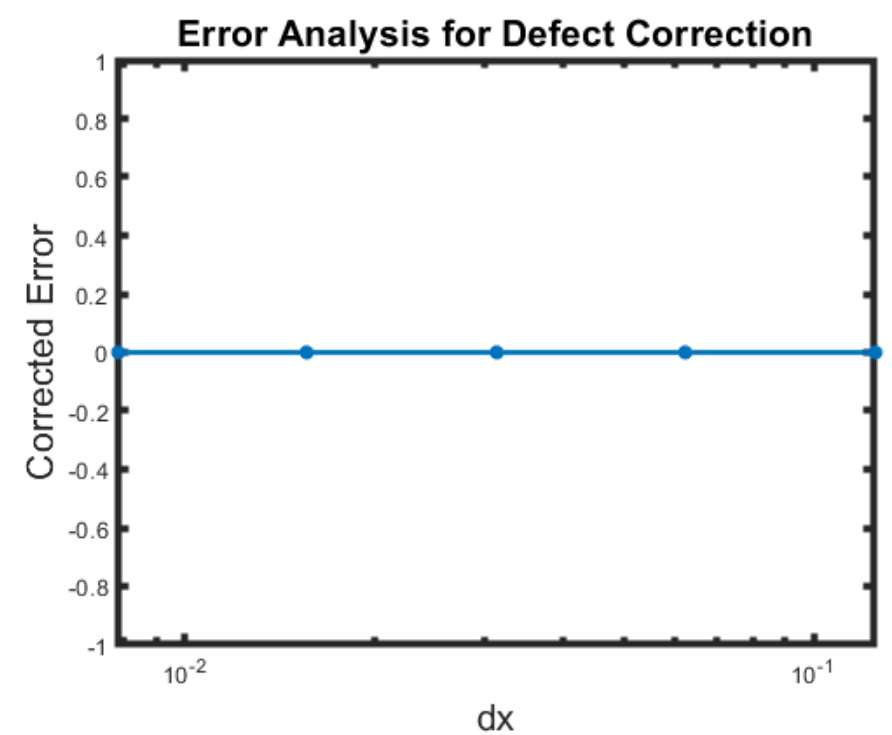

Figure 2.10: Unsteady Burgers' order analysis with exact truncation error 


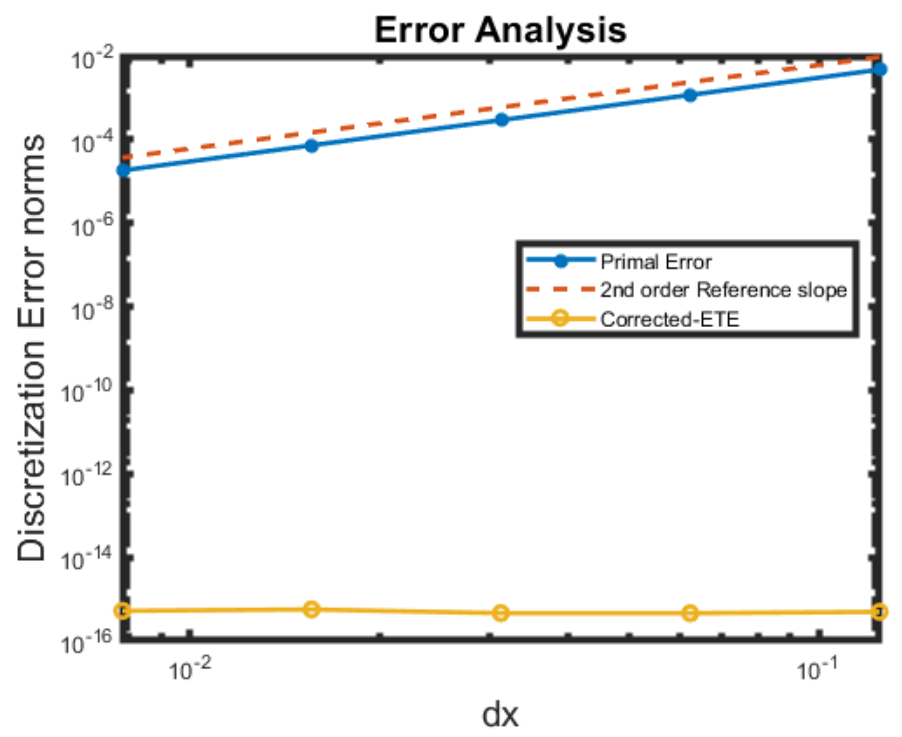

Figure 2.11: Unsteady Burgers' discretization error comparison with exact truncation error

It is uncommon to have an exact solution to complex unsteady problems and hence the estimated truncation error being used as a source term must be analysed. A similar approach can be adopted with the estimated truncation error for defect correction and error transport equation and from Figure 2.12, we see that we get a fourth order accurate corrected discretization error for both ETE and defect correction in comparison to the second order accurate primal discretization error. A mixed order analysis is performed for this case and after solving the full non-linear equation (Eq. (2.32), we see that we get the expected fourth order in space and fourth order in time. The temporal errors are extremely small in comparison to the spatial term and therefore the mixed order term is of importance. 
Chapter 2. Discretization Error Estimation for Unsteady flows using ETE and defect Tejaswini Gautham

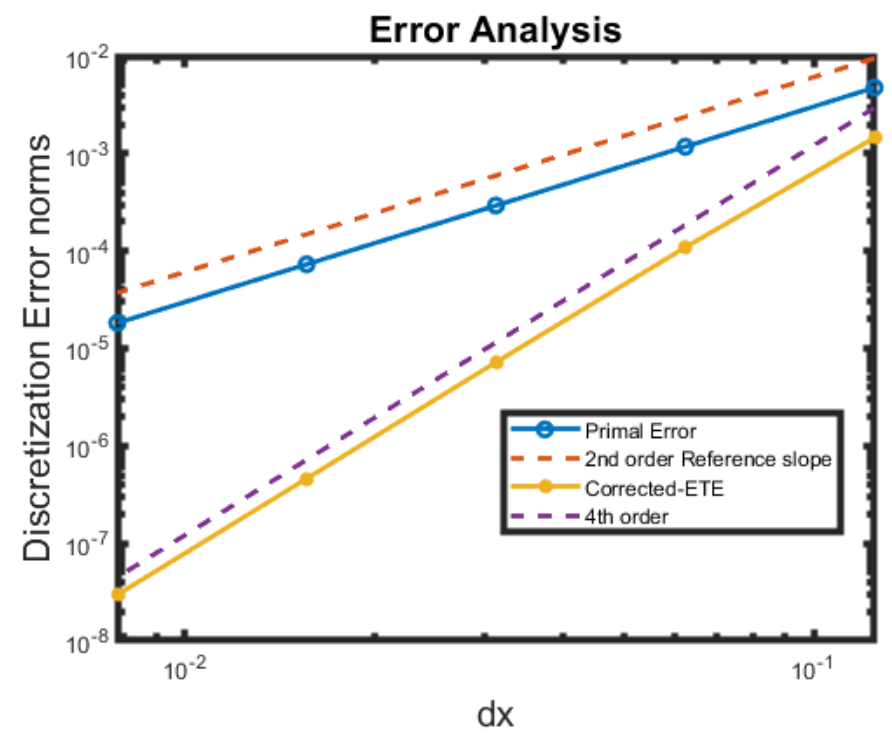

Figure 2.12: Unsteady Burgers' discretization error comparison with estimated truncation error

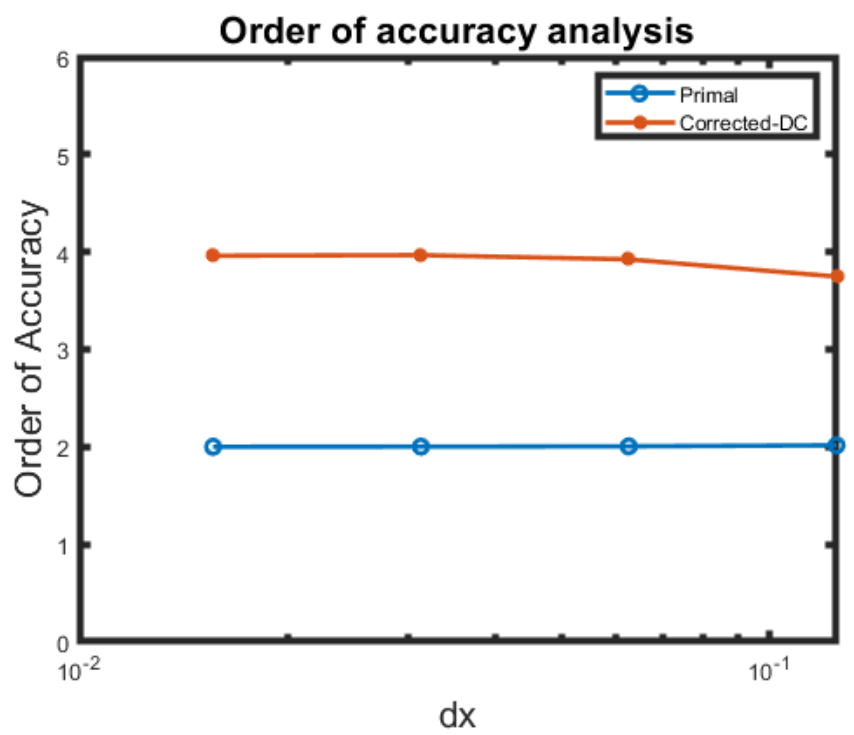

Figure 2.13: Unsteady Burgers' order analysis with estimated truncation error 


\subsubsection{Euler Steady Results}

With exact truncation error as the source term, the same algorithm that was used for steady Burgers' equation is used for the Euler equations and similar results are obtained as shown in Figure 2.14. Here non-linear ETE is used instead of linearized ETE and therefore we get machine precision errors and hence non-linear ETE is more accurate but Non-linear ETE has the same cost as that of defect correct.

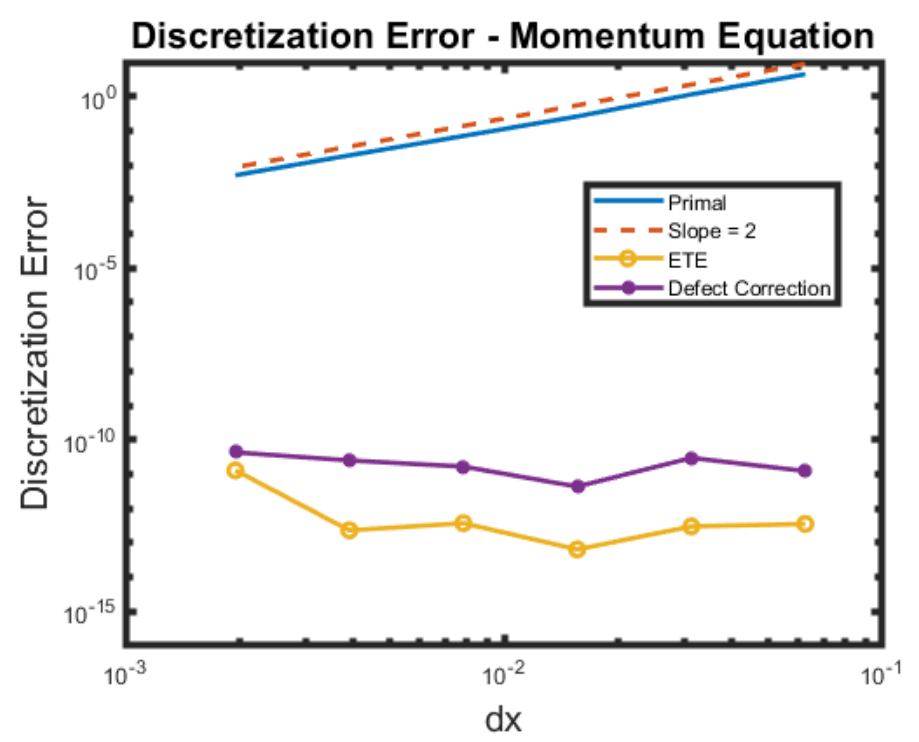

Figure 2.14: Steady Euler discretization error comparison with exact truncation error

A similar approach can be adopted with the estimated truncation error as the source term for defect corrected and error transport equation and we once again obtain fourth order accuracy as seen in Figures 2.15 and 2.16. The error norms are very similar from both approaches and lie on top of each other. 
Chapter 2. Discretization Error Estimation for Unsteady flows using ETE and defect Tejaswini Gautham

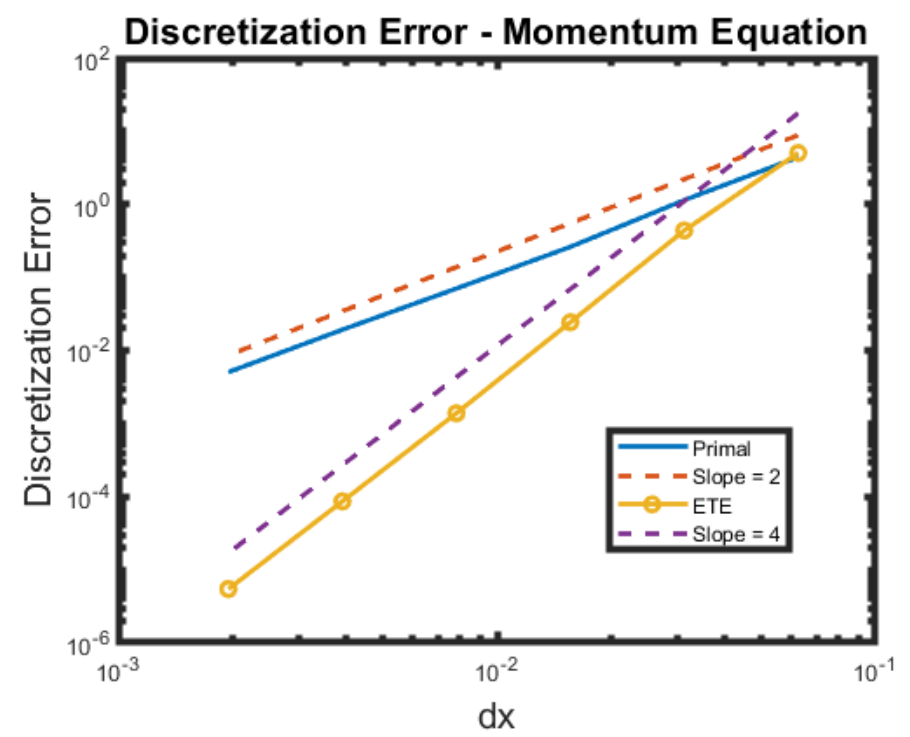

Figure 2.15: Steady Euler discretization error comparison with estimated truncation error

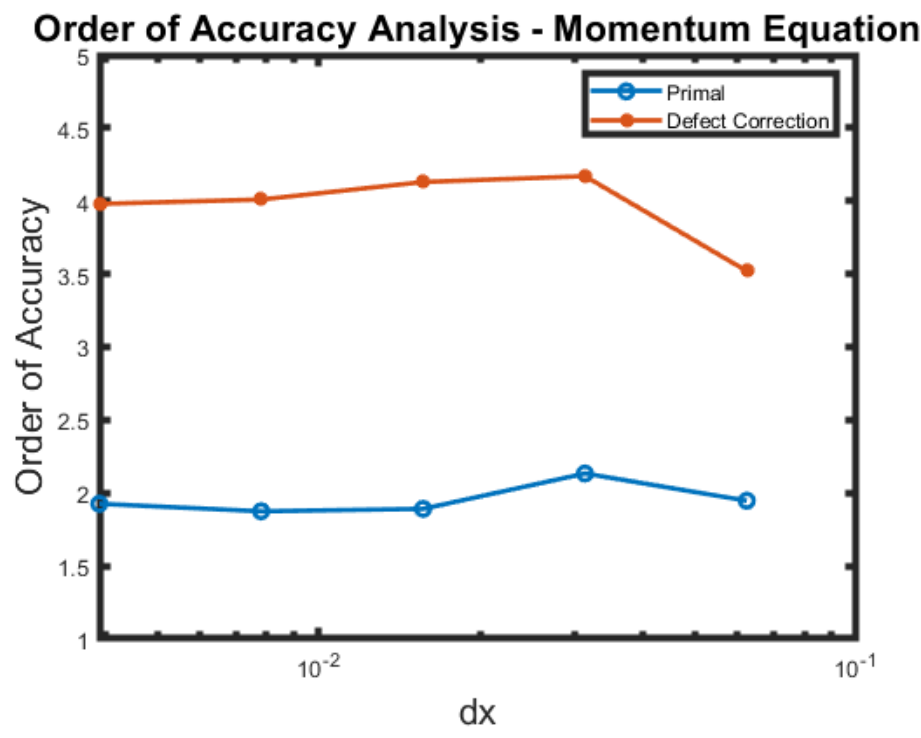

Figure 2.16: Steady Euler order analysis with estimated truncation error

\subsubsection{Euler Unsteady Results}

Using either the manufactured solution as the exact solution or the shock tube solution as the exact solution, with exact truncation error as the source term for defect correction and 
ETE, the same algorithm that was used for Unsteady Burgers' equations is used for Euler equations and the same result is obtained as shown in figures 2.17 and 2.18 and is proof of concept. Burgers' and Euler equations use two completely different discretization schemes but the results remain the same. In spite of the shocks and expansion fans present in the shock tube solution, the exact truncation error is able to capture all the discretization error present in the problem.

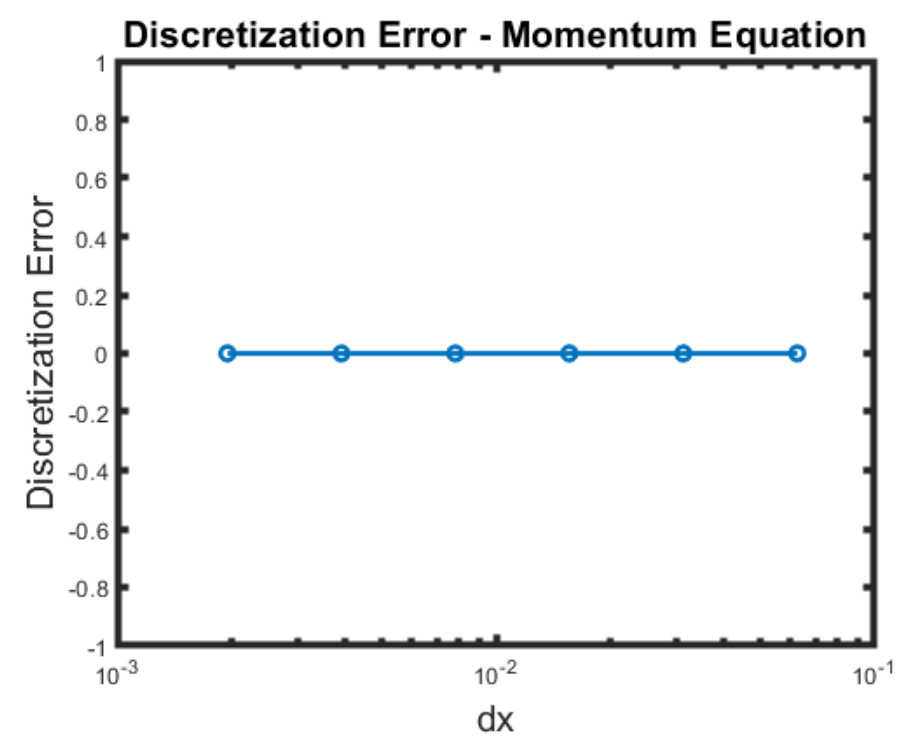

Figure 2.17: Unsteady Euler discretization error comparison with exact truncation error 
Chapter 2. Discretization Error Estimation for Unsteady flows using ETE and defect Tejaswini Gautham

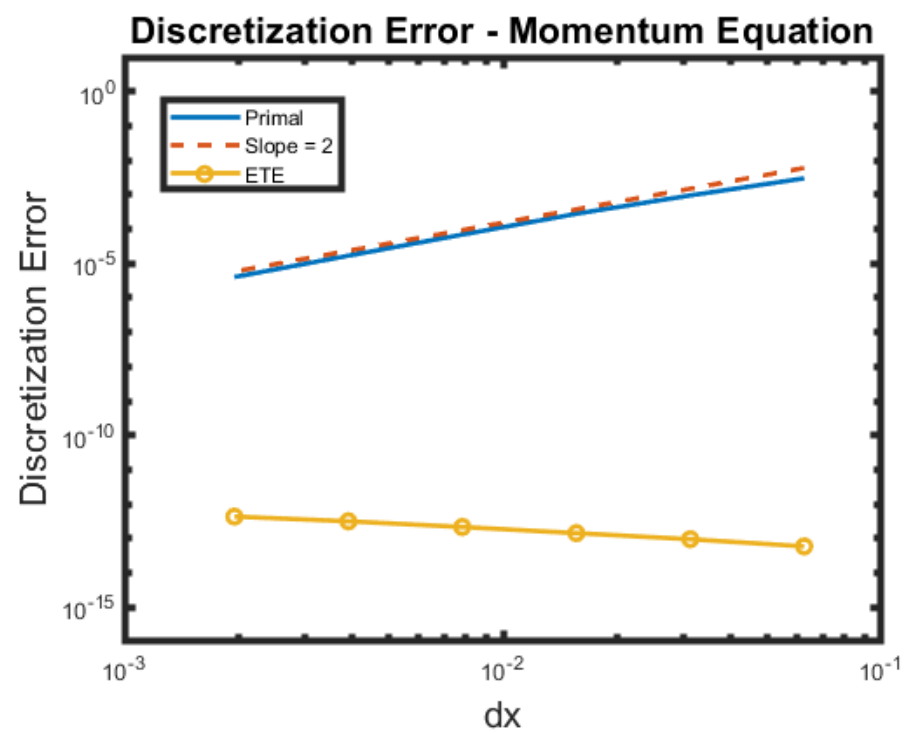

Figure 2.18: Unsteady Euler order analysis with exact truncation error

With the manufactured solution as the exact solution and using estimated truncation error as the source term, defect correction is performed on a first order accurate in time and second order accurate in space discretization. The primal is overall first order accurate and is corrected with a first order accurate truncation error and this leads to significant decreases in errors as compared to the primal as seen in Figure 2.19. Similar results can be obtained by using estimated truncation error as a source term for ETE. As seen in Figure 2.20, the corrected error starts off as first order and finally goes to second for the finer mesh levels. For the sake of brevity, only the momentum equation results have been shown here. 


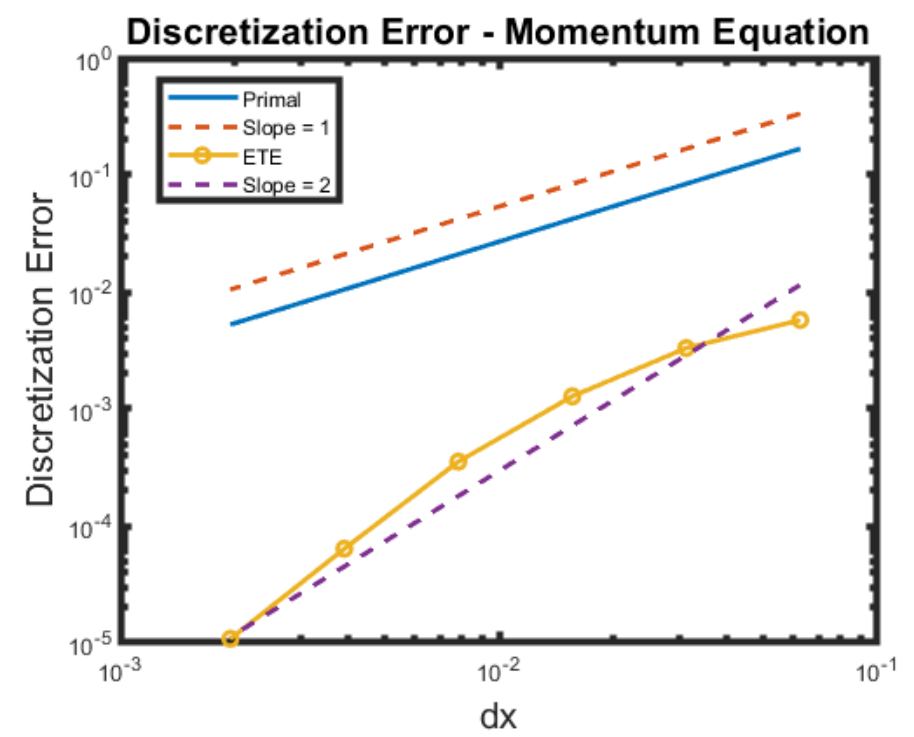

Figure 2.19: Unsteady Euler order analysis with estimated truncation error

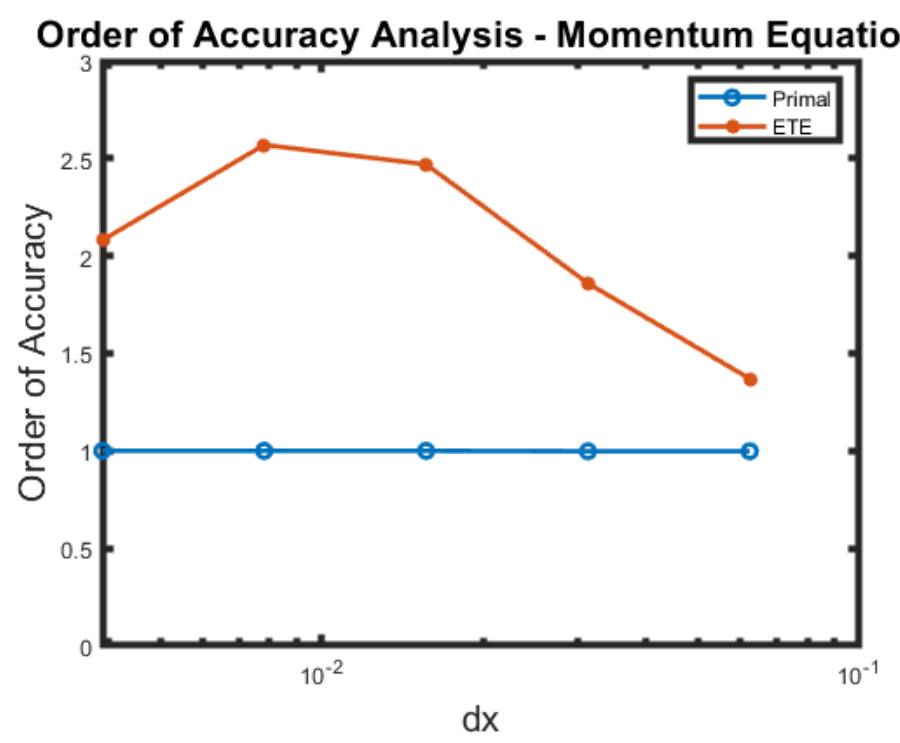

Figure 2.20: Unsteady Euler order analysis with estimated truncation error 
Chapter 2. Discretization Error Estimation for Unsteady flows using ETE and defect Tejaswini Gautham

Chapter 2

correction

\subsection{Conclusions}

In this work, a technique to estimate discretization error is presented. The technique was first presented for the steady case and then extended to the unsteady case. Both, defect correction and the error transport equations were presented and shown to give the better error estimates than the primal problem. In both the steady and unsteady case, either the exact truncation error or its estimate is used as a source term to solve defect correction or the error transport equations. The estimated truncation error is estimated by reconstructing the discrete primal solution and then operating the partial differential equations on it. In the case of steady Burgers' equation and Euler equations, with exact or estimated truncation error as the source term, we get fourth order of accuracy when linearized ETE is used and machine precision errors when non-linear ETE is used. For steady Burgers' equation and Euler equations, for defect correction with exact truncation error as the source term, we see that the corrected discretization error goes to machine precision and estimated truncation error as the source term gives us fourth order accuracy. With regard to unsteady Burgers' equation and Euler equations, with exact truncation error as the source term for defect correction, we get zero error and for exact truncation error as the source term for nonlinear ETE, we get machine precision error. In the case of unsteady Burgers' equation, with estimated truncation error as the source term, we get fourth order of accuracy for both defect correction and ETE. For unsteady Euler, with estimated truncation error as the source term, we see that we get a higher order of accuracy than the primal. Defect correction and non-linear ETE have a similar cost and a similar accuracy of error estimation. Another observation is that non-linear ETE is more accurate than linearized ETE when using exact truncation error as the source term. We get only fourth order accurate error estimates with non-linear ETE but machine precision error estimates with non-linear ETE. However. non-linear ETE is more expensive to compute than linearized ETE. Thus, there is trade-off 
between speed and accuracy. The results were presented for steady and unsteady Burger's equation and Euler equations on structured grids and the corrected discretization error is shown to be of higher order of accuracy than the primal discretization error.

\subsection{Acknowledgments}

This material is partially based on research sponsored by the U.S. Air Force under agreement number FA865019-2-2204. The U.S. Government is authorized to reproduce and distribute reprints for Governmental purposes notwithstanding any copyright notation thereon 


\section{Chapter 3}

\section{Discussion and Conclusions}

Discretization error will always be present in numerical solutions, and this work provides a technique to reduce it significantly and get more accurate estimates of the desired solution. In this work, truncation error is taken to be the local source for discretization error (this has been shown in previous works [1]). It is thus estimated and is used to correct the primal solution to get a better error estimate in defect correction or it is used in ETE to correct the estimated discretization error. Both Error transport equation and defect correction have been applied and are shown to give similar results. The techniques are first implemented on the steady Burgers' equation and are then extended to the unsteady Burgers' and Euler equations. Truncation error estimation is performed for both the steady and unsteady case. For the steady case, the truncation error is only the residual term of the equation. A onedimensional reconstruction of the primal problem is sufficient to estimate the residual. In the unsteady case, the truncation error involves both the unsteady term and the residual. This involves a two dimensional reconstruction in space and time. This is not very straightforward particularly for finite volume discretization as the conservation of the mean is of importance and hence adds additional terms in the truncation error. Both defect correction and error transport equations give similar results and the cost of solving them are comparable (when non-linear ETE is being used). On the whole, we see that the discretization error can be estimated and a better solution estimate can be found. 


\subsection{Future Work}

While discretization error will always be present in numerical solutions, defect correction and ETE provide a manner to reduce the error significantly. In this work, truncation error is shown to be the local source for discretization error and an appropriate driver for both ETE and defect correction. Only explicit schemes have been explored in this work. This can be extended to implicit schemes that are likely to run much faster. Another arena to explore could be turbulence and discontinuities. Turbulence is inherently unsteady and thus would be a potential application of these techniques. Discontinuities can also be unsteady, as seen in the case of the shock tube, and thus would also be a possible application of these techniques. 


\section{Bibliography}

[1] JW Banks, JAF Hittinger, JM Connors, and CS Woodward. Numerical error estimation for nonlinear hyperbolic pdes via nonlinear error transport. Computer Methods in Applied Mechanics and Engineering, 213:1-15, 2012.

[2] Ismail Celik and Gusheng Hu. Single grid error estimation using error transport equation. Journal of Fluids Engineering, 126(5):778-790, 2004.

[3] L Fox. Some improvements in the use of relaxation methods for the solution of ordinary and partial differential equations. Proceedings of the Royal Society of London. Series A. Mathematical and Physical Sciences, 190(1020):31-59, 1947.

[4] Leslie Fox. The numerical solution of two-point boundary problems in ordinary differential equations. Courier Corporation, 1990.

[5] Antony Jameson. Origins and further development of the jameson-schmidt-turkel scheme. AIAA Journal, pages 1487-1510, 2017.

[6] Matthew Kurzen, Tyrone Phillips, Christopher Roy, and Andrew Sinclair. Method of nearby problems for generating exact solutions to $1 \mathrm{~d}$ unsteady and $2 \mathrm{~d}$ steady problems. In 19th AIAA Computational Fluid Dynamics, page 3652. 2009.

[7] W Layton, HK Lee, and J Peterson. A defect-correction method for the incompressible navier-stokes equations. Applied Mathematics and Computation, 129(1):1-19, 2002.

[8] Anna Naumovich, Malte Förster, and Richard Dwight. Algebraic multigrid within defect correction for the linearized euler equations. Numerical Linear Algebra with Applications, 17(2-3):307-324, 2010. 
[9] William L Oberkampf and Christopher J Roy. Verification and validation in scientific computing. Cambridge University Press, 2010.

[10] Victor Pereyna. Iterated deferred corrections for nonlinear boundary value problems. Numerische Mathematik, 11(2):111-125, 1968.

[11] Victor Pereyra. On improving an approximate solution of a functional equation by deferred corrections. Numerische Mathematik, 8(4):376-391, 1966.

[12] Victor Pereyra. Iterated deferred corrections for nonlinear operator equations. $\mathrm{Nu}$ merische Mathematik, 10(4):316-323, 1967.

[13] Tyrone Phillips. Residual-based discretization error estimation for computational fluid dynamics. PhD thesis, Virginia Tech, 2014.

[14] Tyrone Phillips and Christopher J Roy. Defect correction and error transport discretization error estimation for applications in cfd. In 32nd AIAA Applied Aerodynamics Conference, page 2572, 2014.

[15] Y Qin and T Shih. A discrete transport equation for error estimation in cfd. In 40th AIAA Aerospace Sciences Meeting \&3 Exhibit, page 906, 2002.

[16] Lewis Fry Richardson. Ix. the approximate arithmetical solution by finite differences of physical problems involving differential equations, with an application to the stresses in a masonry dam. Philosophical Transactions of the Royal Society of London. Series A, Containing Papers of a Mathematical or Physical Character, 210(459-470):307-357, 1911.

[17] Chris Roy, Curt Ober, and Tom Smith. Verification of a compressible cfd code using the method of manufactured solutions. In 32nd AIAA Fluid Dynamics Conference and Exhibit, page 3110, 2002. 
Tejaswini Gautham

[18] Christopher Roy. Strategies for driving mesh adaptation in cfd. In 47th AIAA aerospace sciences meeting including the new horizons forum and aerospace exposition, page 1302, 2009.

[19] Christopher Roy. Review of discretization error estimators in scientific computing. In 48th AIAA Aerospace Sciences Meeting Including the New Horizons Forum and Aerospace Exposition, page 126, 2010.

[20] Lee Shunn, Frank Ham, and Parviz Moin. Verification of variable-density flow solvers using manufactured solutions. Journal of Computational Physics, 231(9):3801-3827, 2012.

[21] Robert D Skeel. Thirteen ways to estimate global error. Numerische Mathematik, 48 (1):1-20, 1986.

[22] Hans J Stetter. The defect correction principle and discretization methods. Numerische Mathematik, 29(4):425-443, 1978.

[23] F Toro Eleuterio. Riemann solvers and numerical methods for fluid dynamics, 1997.

[24] William C Tyson, Katarzyna Swirydowicz, Joseph M Derlaga, Christopher J Roy, and Eric de Sturler. Improved functional-based error estimation and adaptation without adjoints. In 46th AIAA Fluid Dynamics Conference, page 3809, 2016.

[25] William Conrad Tyson. On Numerical Error Estimation for the Finite-Volume Method with an Application to Computational Fluid Dynamics. PhD thesis, Virginia Tech, 2018.

[26] Kai Kin Gary Yan. Numerical estimation of discretization error on unstructured meshes. PhD thesis, University of British Columbia, 2018. 
[27] PE Zadunaisky. A method for the estimation of errors propagated in the numerical solution of a system of ordinary differential equations. In The Theory of Orbits in the Solar System and in Stellar Systems, volume 25, page 281, 1966.

[28] Xu Dong Zhang, J-Y Trépanier, and Ricardo Camarero. A posteriori error estimation for finite-volume solutions of hyperbolic conservation laws. Computer methods in applied mechanics and engineering, 185(1):1-19, 2000. 